\title{
DOUTRINA.
}

\section{Remarques sur l'abus des Droits em Droit Romain Classique.}

\author{
Alexandre A. Corrêa \\ Professeur de Droit Romain à la Faculté de \\ Droit de l'Université de São Paulo (Brésil).
}

\section{Introduction.}

Les textes du Droit Romain rélatifs à ce que nous appelons l' abus des droits sont nombreux et apparaissent dans les rapports les plus variés soit du droit privé soit du droit public. À notre avis à travers l' examen des differentes espèces il est possible de dégager une conception générale de l' abus telle que les jurisconsultes romains auraient eu et semblable à la nôtre. Le problème est d'interprétation et il pose aussi une question d'ordre plus profonde, à savoir celle de décider si en statuant sur les cas particuliers, le Droit Romain était ou non guidé par des principes.

Or, il nous semble pouvoir répondre affirmativement à la question d'abord pour une raison d'ordre historique: si les Grecs sont reconnus comme les créateurs de la Philosophie pourquoi les Romains n'auraient-ils pas pu avoir eu des idées philosophiques, surtout quand on sait que sous le rapport de la pensée ils ont été les disciples des Grecs? Ensuite, quoique n'ayant pas exprimé de façon claire le principe de l'abus des droits, les Romains, comme toujours, l'ont intuitivement senti; et, d'une façon générale, dans nos rapports intellectuels avec les Anciens il nous a seulement fallu étendre la portée de leurs intuitions.

* (Comunicação debatida e aprovada no Seminário Internacional de Direito Romano, realizado em Perugia (Itália), de 11 a 14 de outubro de 1971). 
Nous soutenons donc que pour l'essentiel les Romains ont clairement senti le besoin de prohiber l'abus des droits quoiqu'ils ne se soient jamais souciés de formuler des principes. Les sources sont connues et elles sont discutées, comme on le sait, sutout à propos du droit de propriété.

Mais, on les retrouve partout, à notre avis, dans les differentes branches du droit public et privé. Au point de vue politique et historique, songeons au passage de la Royauté à la République: le dernier roi de Rome a été déposé à cause de ses abus puisque d'aprés le droit, au point de vue du "jus" ses pouvoirs n'étaient pas limités. Les "mores" seulement imposaient des bornes pour ainsi dire "subjectives" à l'arbitraire du monarche. Le manque de respect à ses devoirs envers le peuple a causé la "révolution" punissant l' abus. L' "Intercessio", d'autre part, que le tribun de la plèbe pouvait opposer à l'activité des consuls trouve sa raison d'être dans l'idée de défense contre des abus d'ordre politique, de même que la responsabilité des magistrats vis-à-vis du peuple.

Mais si nous mettons de coté les questions de Droit Public, si intéressantes soient-elles, en nous limitant au Droit Privé, il n'est pas difficile de brosser les grandes lignes d'un tableau d'ensemble oú l' idée de combat à l'abus du droit joue un rôle considérable.

Pensons, en effet, aux trois "juris praecepta" tout d'abord et en particulier au second, "neminem laedere" ${ }^{1}$ Ensuite, l' "aequitas" est un concept directeur, capable, à tout moment, de faire rentrer dans la sphère juridique les intérêts moraux et économiques les plus variés. L' "exceptio doli", enfin, et pour nous en tenir dans des bornes étroites, cet "everriculum malitiarum omnium" dont parle quelque part Cicéron est l'instrument le plus efficace dans la lutte journalière du Droit contre l'abus.

1. Inst. 1, 1, 3 -; D, 1, 1, 1, 2, UlPIEN 
On pourra sans doute soulever tout de suite contre notre thèse cette objection rédoutable: si on parle de "dolus" il s'agit d'acte illicite et d'acte contraire au droit rendant tout à fait dispensable la notion d' "abusus", située en dehors de la sphère propre du droit. Mais à cela on peut répondre, de façon générale, en disant: la règle de croit n'opère pas à vide; elle a nécessairement un contenu d'ordre moral, politique, religieux ou économique lequel ne doit pas être violé ou "abusé" C'est vrai, d'autre part, que "Non omne quod licet honestum est" Ce texte montrerait la séparation de la morale et du droit, plaçant l' abus en dehors de celui-ci. Toutefois les rapports entre "honestum" et "justum" sont flottants et peuvent toujours changer.

Le fameux texte de Tacite, "Corruptissima republica plurimae leges" 2 parait, en effet, montrer le récul croissant des frontières de la morale au fur et à mesure que le droit voit grandir sa protection à des valeurs situées d'abord au delà de sa portée. En d'autres termes, la réaction contre des abus met en mouvement la force coercitive du droit, la "sanctio" et par là le droit lui-même se perfectionne et se développe.

C'est dire que la notion d'abus se situe à coté de celle de "jus" et la met en mouvement, pour ainsi dire. On ne peut se passer d'une si précieuse notion pour comprendre l' évolution de n'importe quel ordre juridique ancien ou moderne. Nous ne voulons pas nous engager dans la philosophie du droit mais notre thème se situe à une espèce de croisée de chemins.

La loi est inconcévable sans les conditions sociales de sa promulgation et les buts qu'elle poursuit dépassent parfois son texte, sans qu'il soit, d'autre part, jugé possible de tolérer certains comportements nuisibles et apparement légitimes. "Scire leges non est verba earum tenere sed vim et potesta-

2. Annales, 3,27 . 
tem" dit Celse dans ce texte si connu. Mais, l'esprit des lois est trouvé par voie d'interprétation et ce travail est fait à Rome par le préteur aidé des jurisconsultes. Or, ceux-ci donnent leurs avis en fonction d'une conviction laquelle trouve sa source, comme c'est évident, dans les idées régnantes à chaque époque. C'est pourquoi l"Aequitas" se trouve, elle aussi, d'une certaine façon, en dehors du Droit et néanmoins le dirige.

La "Jurisprudentia" à Rome, de même que les édits, fait partie des sources du droit et les magistrats anciens n'átaient pas liés par la loi aussi strictement que ceux d'aujourd'hui. C'est, à notre avis d'ailleurs, une des raisons pour lesquelles la notion d'abus du droit a pu jouer un rôle considérable dans l'évolution du Droit Romain.

La "fraus legi facta", par exemple, illustre de façon saisissante cette verité: l'agent semble obéir à la loi quant à sa forme mais en réalité il viole son esprit et commet une infraction "ad instar" de la violation manifeste.

Réprenons, maintenant, le célèbre "Non omne quod licet honestum est" Là, le Droit semble situé en dehors de la Morale, répresentée par l' "honestum" et, donc, on pourrait objecter qu'un comportement licite ou permis n'est jamais abusif. Mais on peut également dire: le Droit se compose non seulement de la loi mais de la Morale qui l'inspire et en fait partie. Très souvent les juristes romains disent qu'il faut considérer non seulement le "licitum" mais aussi l' "honestum", par application de l' "honeste vivere" 3

Le "Summum jus, summa injuria", finalement, cité par CicÉron dans le "De Officiis" "a le sens indéniable d'une idée générale d'équité, guidant l'exercice d'un droit vers son but véritable, situé parfois au-delà de la simple apparence.

3. v.g., D., 23, 2, 42; D., 50, 17, 197.

4. $1,10,33$. 
L' "exceptio doli" a été justement crée par le préteur avec une portée générale comme a bien vu MAx KaSER, dans son "Manuel" 5

Résumons-nous, pour finir cette Introduction avant d' aborder l'étude des opinions de Riccobono et Bonfante sur les textes classiquement rattachés au thème de l' abus des droits proprement dit. On trouve partout en Droit Romain l'influence de l'idée de répression à des abus. Pourquoi les XII Tables, par exemple, prohibaient-elles dejà le "paterfamilias" de vendre son fils plus de trois fois ("si pater filium ter venum duit filius a patre liber esto") ce qui permit, d'ailleurs, aux praticiens d'inventer, comme on le sait, l' "emancipatio"? " Pourquoi les "manumissiones in fraudem creditorum" étaient-elles défendues "? de même que l' "actio Pauliana" permettait de révoquer les actes pratiqués par le débiteur en fraude de ses créanciers ${ }^{8}$ ? Pourquoi aussi, en droit public, la "provocatio ad populum" admise contre les condamnations capitales prononcées par les magistrats? ${ }^{9}$

Tous ces exemples et encore d'autres qu'on pourrait allonger indéfiniment ("querela inofficiosi testamenti", "bonorum possessio contra tabulas", "indebiti solutio" et "naturalis obligatio", responsabilités du tuteur et du curateur, usucapion, protection à la "bona fides") prouvent à n'en pas douter l'influence persistante, en Droit Romain, de l'idéal d' "Aequitas" incompatible avec l' abus des droits!

C'est, donc, avec une entière conviction que nous nous permettons, pour exprimer notre pensée, de rapporter un texte admirable de Josserand dans son De l'esprit des Droits et de

\footnotetext{
5. KASER, MAX - Derecho Romano Privado (Tr. espagnole), 1 vol. Madrid, $1968-\S 4$, IV (p. 37)

6. G. 1,132 .

7. G. I, 37 et ss

8. D. 42,8 .

9. D. 1, 2, 2, 16; Liv. 2, 8, 2; Cic. De Rep. 2, 31; 2, 37.
} 
leur rélativité - Théorie dite de l" "Abus des Droits" (Paris, 1939, pp. 3-4) Cet auteur cite les grands romanistes français de son époque Georges Cornil et Charles Appleton pour démontrer aux lecteurs modernes que la théorie de l'abus des droits trouve déjà en Droit Romain ses racines. Voici le passage de JOSSERAND:

"Suivant l'observation de Georges Cornil on en rélève de nombreuses traces dans les sources du droit romain; comme le remarque cet auteur il semble que le jurisconsulte Gaïus formulait déjà une théorie générale de l'abus des droits quand, pour justifier l'interdiction des prodigues et la défense aux maîtres de maltraiter leurs esclaves, il proclamait que nous ne devons point mésuser de notre droit: "male enim nostro jure uti non debemus" Et un autre éminent romaniste, M. Charles Appleton a pu écrire que la théorie de l'abus "est si peu moderne que sur elle répose toute l'évolution du droit romain, allant du droit strict vers l'équité"

Et Josserand ajoute: - "L'évolution rémonte jusqu'aux XII Tables pour aboutir à la définition fameuse de Celse, "Jus est ars boni et aequi", ainsi qu'à l'affirmation non moins célèbre de Paul: - "non omne quod licet honestum est" L'odieuse maxime "Dura lex sed lex" qui ne semble pas être vraiment romaine est, en tout cas, complètement fausse en tant qu'expression du droit romain de la bonne époque; elle doit céder le pas à son correctif et à son antagoniste: "summum jus summa injuria", et le droit prétorien, dans son admirable et harmonieux développement, constitue la plus brillante illustration et comme la marche triomphale de la théorie de l'abus"

Nous nous rallions à l'interprétation du grand juriste français dont la pensée nous servira de guide dans l'analyse de la controverse à propos de l'abus des droit en matière de droit de proprieté en Droit Romain classique. 


\section{Étude de Quelques Textes.}

Les textes, même limités au droit de propriété, sont variés; nous choisirons quelques uns d'entr'eux, admettant qu'ils suffisent pour nous aider à prendre position dans la question de l'abus des droits. Commençons par D., 6, 1, 38 de Celse. Il s'agit d'un cas où le possesseur de bonne foi d'une maison, évincé par le "verus dominus" et n'ayant pas droit au remboursement des "impensae voluptuosae" veut éffacer de la paroi des fresques qu'il ne peut pas emporter avec lui; le texte lui dénie ce droit puisque le possesseur n'obtiendra d'autre effet que de causer un dommage à autrui ("nihil laturus nisi ut officias") sans aucun avantage pour lui. Or, on ne doit pas favoriser la malignité ("neque malitiis indulgendum est")

Sauf interpolation, magistralement discutée par RiccoBONO ${ }^{10}$, la condamnation de l'abus, de la "malitia", dans l'exercice d'un droit, semble évidente, aussi bien que le rôle joué par les jurisconsultes romains dans l'acte de mésurer, pour ainsi dire, la légitimité de cet exercice.

En suivant les traces de Scialoja, Bonfante ${ }^{11}$ ne réconnait pas la véritable portée de la pensée de Celse: l'éminent romaniste italien fait une distinction entre des règles générales de droit, d'un côté et, de l'autre, des critères valables pour un cas donné, lesquels le juge doit prendre en considération à cause de l'ampleur de ses pouvoirs. C'est vrai; mais nous savons tous que quoique procédant par des cas particuliers les jurisconsultes romains étaient guidés par des lumières, par une sagesse naturelle dont les intuitions jouaient le rôle de véritables principes implicites.

On ne peut pas méconnaitre le sens du "malitiis non est

10. RIccoвono, La teoria dell'abuso di diritto nella dottrina romana, Milan, 1939, p. 15.

11. Bonfante, Corso di Diritto Romano, II La Proprietà, prémière partie, M1lan, 1966, pp. 351-2. 
indulgendum" sans en même temps rabaisser la jurisprudence romaine à une simple "praxis", inconsciente de ses moyens et de ses buts, isolée dans son particularisme et, par conséquent, sans rapport avec nous, ce qui semble contredit par l'histoire du droit elle-même.

Nous examinons ensuite, d'après Riccobono, le fr. 63 du D., 24, 1. Il s'agit d'une femme mariée ayant, après mariage, joint, "donationis causa", des accessoires au bâtiment du mari. Malgré la règle sur le "tignum junctum" des XII Tables, le Proculéian Nératius, nous dit Riccobono, avait admis "l'actio 'ad exhibendum" moyennant interprétation restrictive du texte de la loi et à condition que la femme puisse retirer quelque avantage de la séparation; autrement elle perdrait les accessoires quoiqu'ils conservassent leur individualité, comme il arrive aux portes, fenêtres et aux marbres.

C'est à dire: l'exercice d'un droit est inadmissible en dehors de quelque profit d'ordre moral ou économique pour son titulaire; la femme ne pourrait évidemment pas agir par méchanceté ou par dépit envers son mari: "De eo quod uxoris in aedificium viri ita conjunctum est, ut detractum alicujus usus esse possit, clicendum est agi posse" RiccoBono ajoute ${ }^{12}$ un texte analogue de Marcellus au $D$., 30, 43, 1 et dans lequel le "jus tollendi" est réconnu au mari sous réserve d'une utilité pour lui et sans dommage pour la femme: "posse eum haec detrahere, quae usui ejus futura sint, sine mulieris tamen damno" C'est toujours la même idée de prohibition d'un abus et dans un texte classique dont l'authenticité est acceptée.

Dans D., 25, 1, 9, “ita enim permittendum est marito auferre ornatum quod posuit si futurum est ejus quod abstulit", la même conception se présente, quoique, cette fois-ci

12. Riccobono, S., La teoria dell'abuso di diritto nella dottrina romana, Milan, 1939, p. 17 . 
le texte soit suspect d'interpolation comme le reconnait d'ailleurs RicсовоNo.

Quant à GaIus, I, 53 (Inst. 1,8,2) ce texte semble nous introduire au coeur même de la prohibition de l'abus des droits, quoique Bonfante ${ }^{1 s}$ juge, comme on sait, de façon contraire, en s'appuyant sur l'idée de ce qu'il appele un motif éthique ("motivo ético") qu'on ne pourrait pas vouloir élever à la catégorie de principe juridique. C'est, là aussi, pour nous, le centre de notre divergence avec l'illustre romaniste: ce motif, pensons-nous, etait présent comme guide et critère des décisions, sans qu'il fut nécessaire de l'ériger en principe d'abord pour le rendre ensuite éfficace; il existait à l'état latent et d'après l'esprit du Droit Romain, se manifestait dans les diverses questions particulières, d'ailleurs si nombreuses qu'on ne peut à notre avis pas nier son existence dont la valeur égale celle d'un principe. On sait que la méthode du Droit Romain classique n'était pas la déduction rigoureuse et abstraite, allant du principe à ses conséquences même lointaines, mais de là on ne peut pas conclure que certains principes, tels que celui contenu dans le "male enim nostro jure uti non debemus" étaient absents, même sous forme implicite, des grandes décisions des jurisconsultes romains. Qu'est-ce, d'ailleurs, qu'un motif éthique sinon l'idée d'une valeur de justice et d'équité, contrôlant l'usage d'un droit déterminé? Ainsi envisagé ce motif joue en Droit Romain classique un rôle comparable à celui d'un principe dans le droit moderne, lequel n'est d'ailleurs pas purement abstrait ou rationnel mais a eu une évolution historique, à partir, surtout, du Droit Romain, dont les solutions ont été généralisées.

Si des idées semblables au "male enim nostro jure uti non debemus" se présentent constamment dans les textes, à côté du "qui suo jure utitur neminem laedit" ne peut-on raisonnablement conclure de là à l'existence en Droit Romain clas-

13. Bonfante, op. cit, pp. 350-1. 
sique d'une règle comparable, par ses résultats pratiques, au principe de l'abus?

Il nous semble légitime de l'affirmer, surtout si l'on s'apperçoit qu'en Droit Moderne, en France par exemple, comme l'atteste JoSSERAND, dans son ouvrage cité, la théorie de l'abus s' est dégagée à partir du début de ce siècle comme affaire de Jurisprudence, offrant, sous ce rapport, une ressemblance frappante avec le Droit Romain classique. N'oublions d'ailleurs pas que Scialoja, lui-même, dont Bonfante parait suivre l'orientation, réconnaissait que son interprétation niant l'existence, en Droit Romain classique, du principe de l'abus des droits n'était pas acceptée par l'unanimité des romanistes de son époque tels que Windscheid, Maynz et De Crescenzio en dehors de Cornil et d' Appleton. Et même anjourd'hui Max Kaser par exemple, dans l'édition espagnole de son Derecho Privado Romano, déjà cité ${ }^{14}$ attribue à l'“exceptio doli" un caractère général de prohibition de l'abus des droits, tandis q'une conception rattachée aux idées de Scialoja et de Bonfante se reproduit chez Grosso ${ }^{15}$

La question est donc controversée, permettant des essais de solution tels que celui-ci, inspiré d'ailleurs par la monographie de Riccobono lequel s'oppose d'une façon éclairée au rejet de Scialoja et de Bonfante en montrant que le droit quiritaire lui-même réprimait déjà l'"abus" en sanctionnant l'interdiction du prodigue (G., 1,53) au nom de l'intérêt de la famille, avant de justifier la mésure par l'intérêt du prodigue lui-même.

La répression, d'autre part, à la cruauté des maîtres, s'inspirait d'un sentiment d'humanité et de charité, grandi sous l'influence de la morale stoicienne, faisant considérer l'esclave plutôt comme "personna" que comme "res".

14. Madrid, 1968 \& 4, IV (p. 37)

15. Grosso, GIUSEPPE, Abuso del diritto a) Diritto Romano in "Enciclopedia del Diritto", Milan, I, 1958, pp. 161-163. 
Donc, tout un ensemble d'idées et de courants historiques éveillait l'esprit des juristes romains aux problèmes des bornes à tracer entre l'exercice normal d'un droit et son usage abusif!

C'est pourquoi nous nous rangeons à côté de Riccobono quant il s'étonne d'entendre Scialoja nier la valeur probatoire du texte de Gaius lequel condamne si clairement le mauvais usage du droit de propriété, eu égard au manque d'intérêt de la part du titulaire et aux conséquences sociales fâcheuses produites par l'abus.

La prohibition de l'abus, en matière de propriété, remarque avec raison le maitre italien, se trouve en germe dans ce texte lequel, ajouté à d'autres, a une grande valeur historique, comme point de départ d'une tendance nouvelle qui se fait jour dans le Droit Romain lequel sous l'action d'idées éthiques et sociales restreint peu à peu le droit quiritaire tout d'abord extrêmement individualiste si on peut le dire ${ }^{16}$ Pour Bonfante et aussi pour Scialoja, au contraire, l'idée d'une limite posée à l'exercice du droit ne signifie rien d'autre que la négation voire la destruction même du droit, puisqu'on soumet les pouvoirs qu'il renferme au contrôle du juge et du juriste, lesquels mésureront les prérogatives du titulaire d'après une logique sans fondement dans les principes positifs de la loi, ayant recours à des motifs d'ordre purement moral ou à des considérations pratiques non prévues par la loi ${ }^{17}$

Ces deux romanistes se posent donc en partisants résolus du "qui suo jure utitur neminem laedit" contre le "male enim nostro jure uti non debemus" préféré par Riccobono et par nous comme l' expression plus authentique du Droit Romain même classique.

16. Op. cit. pp. 19-20.

17. BONFANTE, op. cit. pp. 342-3. 
En matière d'eaux le droit classique offre une réglementation détailleé, due probablement aux conditions géographiques et météorologiques de l'Italie, atteinte par une relative sécheresse, laquelle affecte de façon évidente les bésoins agricoles du pays et fait à notre avis éclater le souci de protéger le bon usage du liquide nécessaire aux plantations en même temps que le gaspillage et l'emploi improductif sont réprimés.

Là, il nous semble rencontrer des exemples frappants du combat à l'abus du droit de proprieté.

Toujours à la suite de Riccobono examinons quelques textes, discutés aussi par Bonfante, dont nous rejetons en partie au moins les conclusions. Le premier de ces maitres fait d'abord ressortir, de façon très ingénieuse, le rapport interne unissant les trois textes du D. 39,3 (1,11-12; 2, 9; 2,5) à l'autre, déjà examiné, de Celse (D. 6, 1, 38) lequel condamnait l'exercice dolosif du "jus tollendi", et mettait en même temps en cause le problème de l'abus du droit.

Riccobono nous éclaire aussi sur l'état de la question au temps des Proculiens et des Sabiniens, les premiers suivant une conception plus évoluée du droit de proprieté, tandis que les seconds étaient plutôt conservateurs et rigides dans leur réconnaissance des prérogatives du "dominus" C'est, en d'autres termes, affirmer implicitement l' existence, chez les classiques, d'une controverse touchant les limites posées à l'exercice du droit, c'est à dire au problème de l'abus.

Tel, du moins, est nôtre avis.

Passons donc à l'éxegèse des textes.

Dans D. 39, 3, 1, 11 Ulpien rapporte l'opinion de Sabinus et de Cassius, selon lesquels chacun, en labourant son domaine, a le doit de retenir l'eau pluviale qui y tombe et aussi celui de faire écouler vers son fond l'eau du voisin sous condition de ne rien entreprendre sur le terrain d'autrui; et la raison donnée est toute empreinte d'équité et de bon 
sens; personne n'est prohibé de jouir d'un avantage quelconque sur le bien d'autrui s'il s'agit non de nuire le voisin mais simplement de rendre sa terre meilleure.

Doctrine qui manifeste la plus pure humanité du juriste romain, doué d'un sens véritablement social de la justice et de la destination authentique du droit de proprieté, qui est d'unir plutôt que de séparer les homens!

Le paragraphe suivant du même fragment condamne, à notre avis, de façon claire et indéniable l' abus du droit, en accordant au propriétaire la faculté de creuser un puits dans son terrain, même en détournant les sources qui fournissaient de l'eau au voisin, pourvu que l'ouvrage soit accompli dans le but utile d'améliorer le fonds et non dans celui de porter dommage au voisin.

Dans son commentaire sur ce texte Bonfante ${ }^{18}$ emploie une dialectique très ingénieuse qui fait honneur sans doute à son intelligence mais ne nous semble pas convaincante: d'accord sur ce point avec la pensée de Scialoja le maître italien nie la portée générale de l"animus nocendi" en disant que l'interdiction est strictement limitée à la matière d'eaux, dont l'usage par les propriétaires voisins est d'ailleurs soumis à un régime spécial.

Le critère limitatif du droit de chacun serait d'ordre objectif, celui de l'utilité, indépendamment de l'"animus" du propriétaire. Ainsi, même avec intention de nuire je peux porter dommage à mon voisin s'il résulte quelque utilité pour mon terrain en conséquence de l'ouvrage construit; et réciproquement, même sans "animus nocendi" le dommage fait à autrui est inadmissible s'il ne produit aucune utilité pour celui qui l'a causé.

La remarque est frappante et semble anéantir la portée

18. Op. cit., pp. $355 / 6$. 
du concept d'"animus nocendi" mais on peut répliquer ceci: le manque d'utilité propre est presque toujours lié à l'esprit d'émulation. D'ailleurs, là ou le contenu économique du droit de proprieté disparait, à savoir l'utilité, le droit lui-même s'éteint: l'"animus nocendi" semble implicite, sous forme de faute presumée dans l'exercice sans utilité, voire abusif. Nous réconnaissons la difficulté soulevée par Scialoja-Bonfante, mais elle ne nous semble pas insurmontable si on admet que l'animus nocendi" est fréquernment associé au mauvais usage du droit. Quant à la question de la portée générale ou spéciale des restrictions examinées, nous renvoyons à notre réponse, suggerée à la page 7 du présent travail.

Dans D. 39,3 h. t. 2,9 la même pensée se présente à propos d'un cas analogue, quoique cette fois-ci il s'agisse de décider lequel des deux propriétaires doit supporter un dommage causé non pour obtenir une utilité mais pour éviter un autre dommage. Le texte enseigne qu'un sacrifice patrimonial peut être imposé à mon voisin s'il s'agit pour moi d'éviter un dommage à ce qui m'appartient. Ce serait une espèce de légitime défense du droit de proprieté; d'autre part une haute pensée de solidarité entre voisins semble renfermée dans les paroles du jurisconsulte Paul, lequel éclaircit d' ailleurs en passant le sens du verbe arcere: “aquam' enim arcere hoc esse curare ne influet"

Dans D. 39,3, h.t., 2,5 finalement, nous lisons le célèbre: "Haec aequitas suggerit, etsi jure deficiamur" devenu proverbial. Le cas envisagé est celui d'une digue située dans le fonds du voisin et rompue par la force de l'eau; je peux obliger le voisin à réfaire la digue, puisque ça m'est utile sans lui être nuisible: "qui factus mihi quidem prodesse potest: ipsi vero nihil nociturus est".

Pour Bonfante, nous le savons déjà, la généralisation

19. D., 39, 3, h.t. 2,5 . 
de ce principe conduirait tout droit à l'abolition du droit de proprieté. Nous ne pensons de la sorte, par les raisons déjà indiquées, mais nous ne pouvons nous étendre davantage sur un sujet si passionant et si vaste sans dépasser les bornes tracées par le règlement du "Séminaire" quant au maximum de pages à écrire.

C'est la raison pour laquelle nous finissons ici notre communication sans discuter le seul texte du Digeste qui emploie le terme "aemulatio" (D 50, 10, 3,), ni les admirables remarques de Bonfante sur le sens qu'a pris le mot au Moyen Âge sous la plume des glossateurs.

En cherchant à prendre position, nous espérons avoir l'honneur de discuter notre point de vue avec les illustres collègues romanistes présents au Séminaire ${ }^{20}$

20. Il nous a fallu être à Rome pour lire dans l'édition récente des Istituzioni di Diritto Privato Romano, du Prof. EdUardo VolterRa (Edizioni Ricerche, Roma, 1967) des paroles qui expriment de façon très exacte la pensée que nous défendons. C'est la raison pour laquelle nous voulons les citer et aussl en hommage à l'illustre romaniste italien, lequel, d'ailleurs, a été disciple de Bonfante. Voici le texte du Prof. Volterra, qui nous parait d'importance décisive: “... Gli antichl giuristi pur non avendo formulato teoricamente alcun principio generale in materia, sentivano, con acuta sensibilità, l'importanza sociale dell' esercizio della proprietà cosi come di ogni altro diritto e l'esigenza che non fosse giuridicamente tutelato tale esercizio quando fosse anormale, cioè contrario al fine sociale ed economico dell' ordinamento gluridico" (op. cit., pp. 302-305) 